\title{
Gold is going forensic
}

\author{
Ahmed A. Mohamed \\ Published online: 20 April 2011 \\ (C) The Author(s) 2011. This article is published with open access at Springerlink.com
}

\begin{abstract}
Not long ago, forensic science was mainly focused on fingerprint detection. With the advance in science and technology, forensics has become an increasingly interesting scientific field to explore, and gold is playing an increasingly important role in this area. Gold compounds and gold nanoparticles contribute to fingerprint detection and illicit drug testing. Fingerprints can be detected on diverse suspected articles after being covered with gold followed by another metal such as zinc utilizing vacuum metal deposition technique. An important advantage of using gold in forensic science has been the long-term storage of developed fingerprints due to its inert nature in addition to the high selectivity and the sensitivity of gold nanoparticles. Gold also forms microcrystals with cocaine and heroin of identifiable morphology.
\end{abstract}

Keywords Gold · Forensic · Fingerprints

\section{Introduction}

Gold plays an important role in several applications from industrial to medical. Recently, gold compounds have been used as a "pharmacy on a chip," in which micro doses of drugs are encased inside a thin gold covering on an electronic chip that is implanted in the body. As a chemical catalyst, gold plays an important role in new environmental applications, such as pollution control of mercury emissions and in fuel cells. Several interesting real-world optical applications of gold resulted in marketable optical devices [1-7]. Gold is emerging in a new application: gold is going

\footnotetext{
A. A. Mohamed $(\square)$

Department of Chemistry, Texas A\&M University,

College Station, TX 77843, USA

e-mail: amohamed@chem.tamu.edu
}

forensic! The application of gold in this interesting area of research represents a major step forward in forensic science and places gold compounds in the courtroom.

Gold was used in forensic odontology, which deals with the application of dental science to the law [8]. The examination of dental evidence and dentures was reported as early as 2500 B.C., when two molars linked together by gold wire were found in a tomb in Egypt. Gold in particular and the techniques associated with it provide the answer to some forensic questions: Is the white powder found in possession of the criminal cocaine or heroin? Are the fingerprints left at the crime scene those of a smoker?

There are several areas of forensic science to investigate: latent fingerprint development, illicit drug identification, alcohol measurement in drunk drivers, explosives detection, nerve gas detection, saliva detection, inorganic pigments identification in hit-and-run car accidents, and many more [9-11]. Coordination chemistry plays a major role in forensic chemistry progress by providing complexes that, for example, luminesce in contact with latent fingerprints under laser source, formcolored complexes or microcrystals when mixed with illicit drugs that can be easily identified under a microscope, and complexes, such as potassium permanganate and potassium dichromate, that change color upon reduction in the presence of alcohol. Other inorganic complexes, such as blue copper phthalocyanine, are used as crime markers in car paint. Moreover, silver nitrate or silver nitrate coupled with other metal nitrates is used in the detection of $\gamma$-hydroxybutyric acid (GHB), known as "date-rape" drug [12]. GHB in the presence of some metal nitrates forms crystals with various differences in size, crystallization rate, and color.

The recent expansion in nanotechnology is expected to vastly improve the scientific methodology involved in crime solving. The real contribution of nanotechnology in forensic science research is still limited [13]. The last 
few years have witnessed a growing interest in forensic science applications of gold. The interest in metal nanoparticles and in gold in particular is due to its high stability towards oxidation and the stability of developed fingerprints over time. Gold proved to be an effective metal in fingerprint detection and illicit drug presumptive tests.

\section{Illicit drugs detection}

Some of the most commonly abused drugs are marijuana, amphetamines, barbiturates, cocaine, opium alkaloids, synthetic opioids, gamma-hydroxybutyrate, and ketamine. Cannabis, available as "hash" or "hash oil," is a tobaccolike greenish or brownish material made of the dried flowering tops and leaves of the Cannabis plant. Cocaine (hydrated) is a fine white or off-white powder which is extracted from the leaves of the coca plant. Crack (anhydrous) is cocaine that has been treated with ammonia or sodium bicarbonate and looks like small flakes or rocks. Heroin is a white powder processed from morphine, a naturally occurring substance from the opium poppy plant $[14,15]$.

\section{Presumptive tests for illicit drugs}

Presumptive tests are fast screening procedures that usually consist of two or three independent tests. They are designed to provide a sign of the presence or the absence of drug classes in the test sample and quickly eliminate negative samples [16-18]. There are two main tests that are used in illicit drugs detection, color-generating chemical spot test, and microcrystal test. Color tests are usually the simplest and fastest chemical test that a forensic scientist can apply to a sample with a small quantity and high sensitivity. Color-generating chemical spot tests will not identify a specific substance, but can be used to distinguish classes of drug which react in the same fashion. Microcrystal tests utilizing gold for the analysis of drugs have been used since the 1800s [19]. Crystals formed from the reaction of the drug with a chemical reagent are identified based on their morphology when observed under polarized light microscopy. Although microcrystal tests are quick, simple, and extremely sensitive for the identification of substances, the chemical identity of the crystals is rarely known. There are some structural studies on the crystals formed from this test.

\section{Microcrystal test using gold}

A few metal salts have been used in the microcrystal test. Mercury(II), gold(III), and platinum(IV) salts are used to detect various alkaloid drugs, and they form microcrystals of characteristic color and morphology. Cocaine, heroin, morphine, and codeine are identified by microcrystal test with $\mathrm{H}\left[\mathrm{AuCl}_{4}\right]$. This test involves the addition of a $5 \% \mathrm{w} / \mathrm{v}$ solution of chloroauric acid in water $\left(\mathrm{HAuCl}_{4} \cdot 3 \mathrm{H}_{2} \mathrm{O}\right)$ to a dilute aqueous acidified solution of suspected cocaine, followed by the observation of the resulting crystals microscopically of the envisaged salt (L-cocaine) $\left[\mathrm{AuCl}_{4}\right]$ as shown by X-ray crystallography $[20,21]$.

Treatment of cocaine with the gold reagent ( $\mathrm{HAuCl} 4.3 \mathrm{H} 2 \mathrm{O})$ gives square, yellow crystals upon heating in a water bath. Cocaine with the gold reagent gives square, yellow crystals with heat on a water bath [22]; however, with heroin, large, yellow needles are obtained. Microcrystal tests can be performed on the racemic mixtures of all four cocaine diastereoisomers and on $(-)$-cocaine and $(+)-$ pseudococaine. Three to five percent acid gold chloride in $25 \%$ acetic acid is used as a precipitating reagent (Fig. 1). Cocaine is the only one of the four diastereoisomers to give a crystalline precipitate with the gold reagent [23].

\section{Microcrystal tests for optical isomers of amphetamine and methamphetamine}

The sample powder is treated with one or two drops of 5\% $\mathrm{NaOH}$ solution, and immediately, a drop of $5 \% \mathrm{HAuCl}_{4}$ in $\mathrm{H}_{3} \mathrm{PO}_{4}$ is added. Both D- and L-amphetamines give identical microcrystals, resembling long yellow rods or coarse needles and long narrow blades. The way to distinguish them is to form the racemate, which gives different crystals. Some of the unknown sample powder is added to a small amount of known D-amphetamine salt in one cavity and to a small amount of L-amphetamine salt in another, followed by adding the gold reagent. The mixture that is $(\mathrm{D}+\mathrm{D})$ - or $(\mathrm{L}+\mathrm{L})$-amphetamine will give the long yellow rods while the (D $+\mathrm{L})$-amphetamine racemate gives at first "oily" drops then colored platy crystals [16, 18, 24].

D-Methamphetamine tested with $5 \% \mathrm{HAuCl}_{4}$ in $\mathrm{H}_{3} \mathrm{PO}_{4}$ gives "V" blades with one side shorter than the other. These develop very rapidly if the test is done directly on the dry sample. L-Methamphetamine gives single, crossed blades and "V" blades. D,L-Methamphetamine forms single and "X" blades [16].

\section{Structure of gold cocaine}

Cocaine in blood, after storage, can undergo monohydrolysis to yield benzoyl ecgonine or methyl ecgonine (ecgonine methyl ester), which ultimately degrade to Lecgonine $[25,26]$. The degradation product L-ecgonine has been used as a marker for recent cocaine use [27]. The 
Fig. 1 Photomicrographs of $(-)$ - and $( \pm)$-cocaine crystals obtained with $\mathrm{H}\left[\mathrm{AuCl}_{4}\right]$ in acetic acid [23]
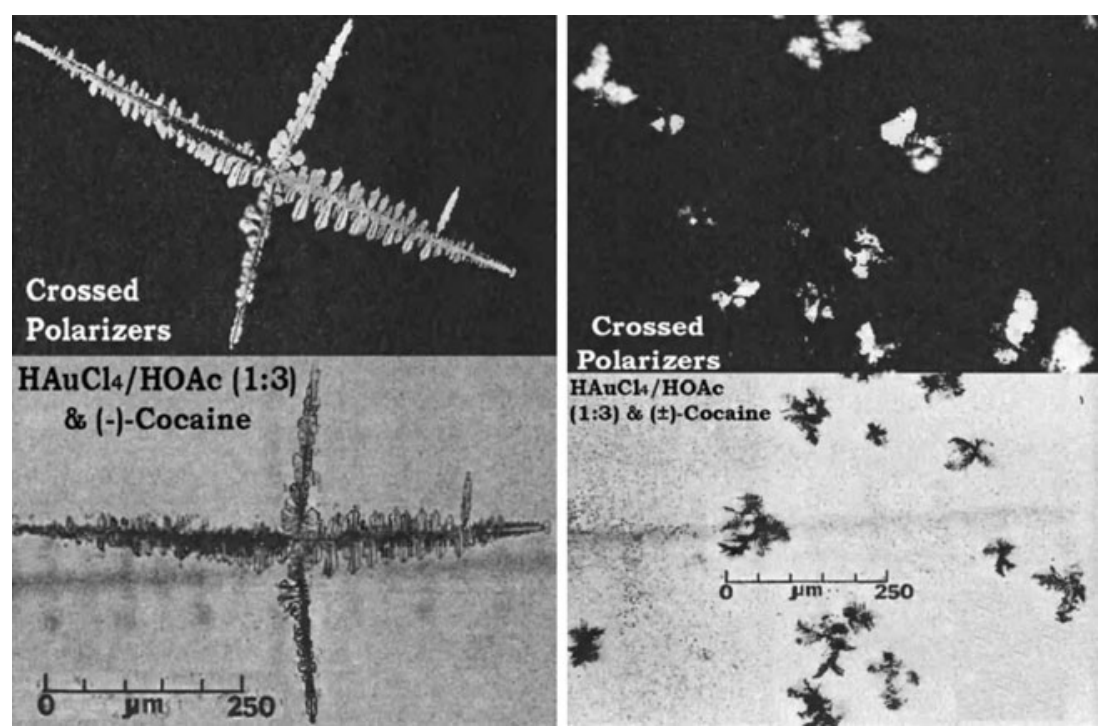

structure of the hydrated gold(III) tetrachloride salt of Lecgonine, (L-ecgonine) $)_{4}\left(\mathrm{H}_{3} \mathrm{O}\right)\left[\mathrm{AuCl}_{4}\right]_{5} \cdot 6 \mathrm{H}_{2} \mathrm{O}$, shows seven water molecules; one of them is protonated. The anhydrous salt has a cation-anion ratio of $1: 1$, (L-ecgonine) $\left[\mathrm{AuCl}_{4}\right]$ $[20,21]$.

Both the hydrate and the anhydrous salts of the same toxicologically important marker for cocaine show that the cation and anion are in close proximity to each other, as was found in the gold(III) tetrachloride salt of L-cocaine. The asymmetric unit contains four monopositive N-protonated L-ecgonine cations surrounded by five $\left[\mathrm{AuCl}_{4}\right]^{-}$anions. For charge balance, one of the seven water molecules has to be a hydronium ion or one of the hydroxyl groups of the cations has to be diprotonated $[20,21]$.

\section{Chemistry of latent fingerprints}

Latent fingerprints consist of a mixture of natural biological secretions [28]. These are typically used for personal identification, and their detection still ranks among the best sources of evidence for crime investigation. New forensic research areas in fingerprint technology have explored interesting chemicals and methods for the recovery of latent prints on various surfaces. These research areas have notably improved the efficiency of forensic science in person identification. These conventional techniques are quite effective in the recovery of latent prints on wet surfaces, surfaces contaminated with body fluids, fabrics, human skin, and porous surfaces.

An average fingerprint residue weighs approximately $0.1 \mathrm{mg}$, of which $98 \%$ to $99 \%$ is water that evaporates over time. This evaporation leaves behind roughly $1 \mu \mathrm{g}$ of residue of which is essentially one-half inorganic materials such as
$\mathrm{NaCl}$ and one-half complex organic materials [29]. The water-soluble portion of the fingerprint deposit is composed of eccrine secretions like salts and amino acids. The waterinsoluble portion is composed of water-resistant proteins and nonpolar lipids.

\section{Detection of fingerprints}

A variety of techniques have been used to enhance the visibility of latent fingerprints. Ninhydrin was first described by Siegfried Ruhemann in 1910 [30]. Ruhemann observed that the ninhydrin reacts with skin and amino acids to produce a purple product. Ninhydrin treatment provides excellent contrast of fresh fingerprints on white paper. The problems of contrast and visualization of weak latent fingerprints have been solved by the treatment of ninhydrin-developed prints with metal salt solution of $\mathrm{Zn}$, $\mathrm{Cd}$, and $\mathrm{Hg}$. Strong room or low temperature luminescence was observed with prints developed with $\mathrm{Zn}(\mathrm{II}), \mathrm{Hg}(\mathrm{II})$, or $\mathrm{Cd}(\mathrm{II})$ ninhydrin solution. Other reagents such as 1,2indanedione and 5-methylthioninhydrin (5-MTN) are close analogues of ninhydrin and react with amino acids in a similar fashion. The development of 5-MTN-treated fingerprints requires heat and humidity, much the same as ninhydrin development. On treatment with a zinc salt, the 5-MTN-developed fingerprint color changes from purple to pink [11].

The cyanoacrylate or superglue fuming method is highly effective for developing latent fingerprints on non-porous substrates. The rapid polymerization on the fingerprint residue results in a white polymer coating that enables print visualization. Metal oxide, sulfide, and selenide powders have been utilized in fingerprint technology such as titanium oxide, zinc oxide, iron oxide, europium oxide, 
molybdenum disulfide, and cadmium sulfide and selenide. Some fingerprint powders may pose a health threat, and the application of these methods in routine casework requires the consideration of occupational health and safety issues [9-11].

\section{Silver physical developer}

The silver physical developer deposits silver on latent fingerprints on porous surfaces [31]. The silver physical developer solution contains silver ions and ferrous ion as the principal components, citric acid and ferric ions as the set of chemicals that suppress the formation of spontaneously formed colloidal silver particles, and a cationic and non-ionic surfactant.

$\mathrm{Ag}^{+}+\mathrm{Fe}^{2+} \rightarrow \mathrm{Ag}+\mathrm{Fe}^{3+}$

Most paper is alkaline due to calcium carbonate filler. When wet, it is basic and it will turn black when dipped in a silver nitrate solution and then dry to a brownish-black color. Alkaline paper must be neutralized by acid before the silver physical development step. The silver physical developer is used to develop the water-insoluble components in the fingerprint.

\section{Gold nanoparticles in fingerprint detection}

Several methods for the development of latent fingerprints are already available. The integration of nanotechnology with these detection methods offers selectivity, enhanced contrast, and higher sensitivity [32]. These nanoparticles are usually either adsorbed electrostatically or their surface is specifically modified to increase affinity for the components of sweat. An important advantage of using gold nanoparticles for this purpose has been the high sensitivity, selectivity, and the long-term storage of developed fingerprints due to their inert nature.

Fig. 2 Fingerprint development with MMD, SMD, and singlemetal nanoparticle deposition (SND). $H Q$ hydroquinone, $A g P D$ silver physical developer
Multi-metal deposition, single-metal deposition, and single-metal nanoparticles deposition

Solution multi-metal deposition (MMD) is a two-step wet chemical process for the detection of latent fingerprints which involves gold and silver. The procedure involves immersing the fingerprint in an aqueous gold solution at low $\mathrm{pH}$ values followed by treatment with physical developer for contrast enhancement (Fig. 2). The physical developer solution contains silver ions, and in the presence of a reducing agent, the ions are reduced to silver metal on gold nucleating sites. MMD is used for visualizing latent fingerprints on a range of porous and non-porous surfaces [32-39].

At low $\mathrm{pH}$ values, the negatively charged gold colloids are electrostatically attracted to the positively charged molecules in the fingermark. Due to the small size of the colloidal gold, it is difficult to obtain a good contrast in order to observe the detected fingerprints. Consequently, it is necessary to enhance the results with the deposition of a second metal onto the surface of gold such as silver in the presence of reducing agent. Each gold aggregate acts as a growing site for the silver particles.

In single-metal deposition (SMD) technique, the silver/ reducing agent bath used in the MMD procedure is replaced with one single gold/reducing agent bath for enhancement. Gold colloids deposited on fingerprints serve as catalysts for the precipitation of metallic gold from the gold/reducing agent solution, thus increasing the size of the gold colloids. The sensitivity and selectivity of SMD procedure are on par with those obtained using MMD [35].

In a trial to minimize the number of baths, a modification of the MMD method was proposed to eliminate the silver physical developer step for contrast enhancement. This was utilized by taking advantage of the molecular recognition mechanisms by functionalizing the gold nanoparticles with a molecular host able to bind itself to gold while keeping the ability to trap molecules in solution. Cyclodextrins, used as hosts for large molecules such as dyes, were chosen as they can be easily chemically

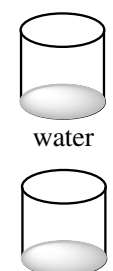

water

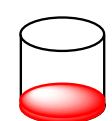

gold-glucose

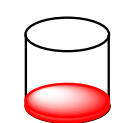

gold-citric

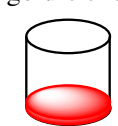

colloidal gold

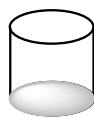

water

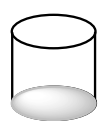

water

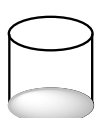

water
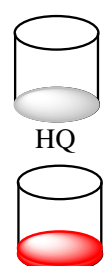

gold- $\mathrm{NH}_{2} \mathrm{OH}$
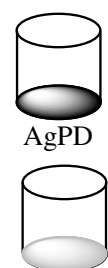

water

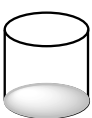

water single-metal

deposition

(SND) 

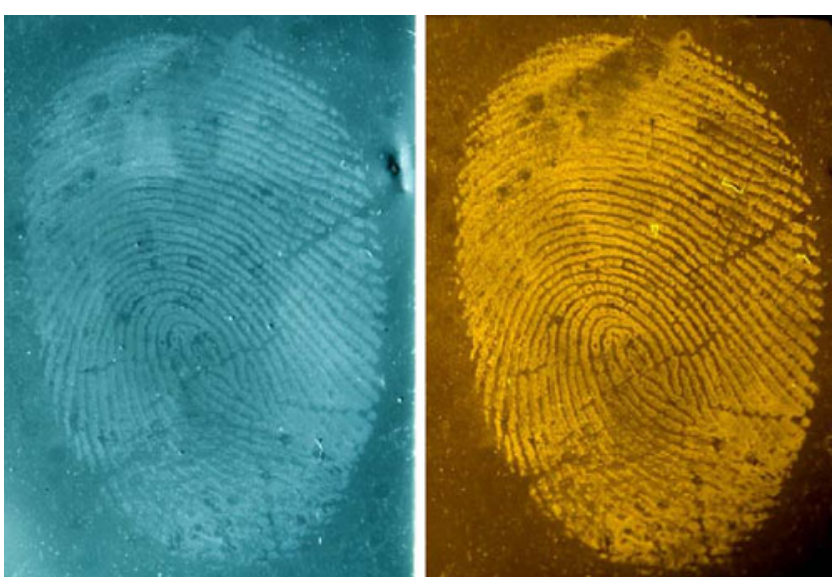

Fig. 3 Latent fingerprint deposited on a black polyethylene bag after the deposition of $\mathrm{ZnO}$. The left picture was observed under a blue light. The right picture was observed under a 300-400-nm luminescence excitation light [34]

modified to offer gold-binding abilities. Detailed fingerprints with good contrast were obtained [32].

A one step, single-metal nanoparticle deposition method for detecting latent fingerprints was performed on several non-porous items in a wide $\mathrm{pH}$ range $(2.5-5.0)$. This method produces reddish fingermarks after development. Gold nanoparticles, synthesized using a reducing agent such as $\mathrm{NaBH}_{4}$ in the presence of glucose as stabilizing agent, were used for latent fingerprints detection [33]. This procedure minimizes the number of baths used for fingerprint development (Fig. 2).

A report utilized the MMD technique to detect fingerprints on black and non-porous surfaces in which zinc oxide can be deposited on gold nanoparticles in aqueous solution and applied to fingermarks (Fig. 3). The improvement of the multi-metal deposition was achieved by obtaining luminescent fingerprints with very good contrast and details. This method comprises a great step forward since it increases the sensitivity of detection on black
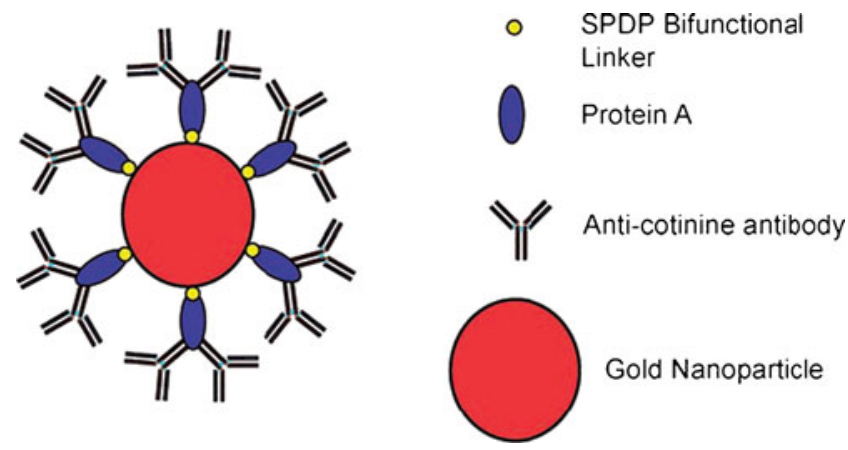

Fig. 4 Representation of the antibody-nanoparticle conjugates. The conjugates are formulated by depositing protein $\mathrm{A}$, which acts as a biological linker to orientate the anticotinine antibody on the gold particle surface. SPDP $\mathrm{N}$-succinimidyl 3-(2-pyridyldithio)propionate [40] surfaces and eliminates the background color, which was one of the main disadvantages of the MMD or SMD techniques $[34,35]$.

The relationship between the thiol chain length and fingermark enhancement was studied. Gold nanoparticles stabilized with tetradecanethiol (Au-NPs-C14), decanethiol (Au-NPs-C10), and octadecathiol (Au-NPs-C18) were synthesized. Sebaceous fingerprints on paper strips were immersed in the different gold nanoparticle solutions, followed by silver physical development. SEM images of the silicon surfaces with fingerprints that had been treated with the different gold nanoparticles showed the following order of aggregation: $\mathrm{C} 18>\mathrm{C} 14>\mathrm{C} 10$ [36].

A one-step method was proposed for the detection of latent fingerprints by in situ production of gold nanoparticles from solutions of $\mathrm{H}\left[\mathrm{AuCl}_{4}\right]$, exploiting the components of human sweat as the sole reducing and binding agents. The components of sweat (lecithin and KI) which were found to be responsible for the formation of gold nanoparticles were then used to develop a bio-ink on paper and polymer [37].

\section{Metal nanopowders}

Fingerprint powdering remains the most commonly used technique for the detection of fingerprints on non-porous surfaces at the crime scene [38]. The technique relies on powder adhering to moisture and oily components in fingerprint ridge deposits. The effectiveness with which the powder adheres to the ridges depends on the size and shape of the particles. Gold nanoparticles could be used in powder form to develop latent fingerprints on nonporous surfaces. It was proposed that oleylamine stabilized gold nanoparticles deposit onto fingerprints due to the lipophilic interaction with the fatty components in fingerprint ridges [39].

The reduction of gold(III) by tin(II) has been known to produce the purple of Cassius, which generates enamel colors from pink to maroon for glass and ceramics. There are many factors that control the size of the nanoparticles such as temperature, $\mathrm{pH}$, and agitation. The control of these factors will result in gold particles of the desired size and color. The gold nanoparticles synthesized from this redox

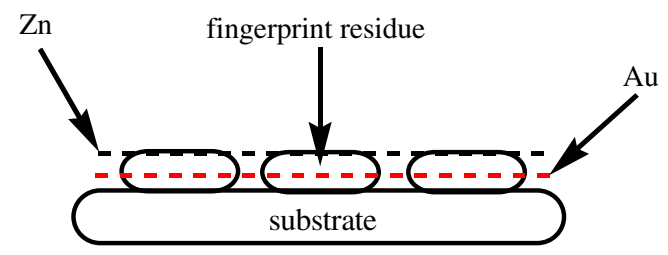

Fig. 5 Vacuum metal deposition procedure on latent fingerprint 
reaction show promising results in fingerprint detection (Mohamed, unpublished results).

\section{Using fingerprint for identification of drug metabolites}

Modified gold nanoparticles were used to detect cotinine, a metabolite of nicotine. The multiple anticotinine antibodies were functionalized with gold nanoparticles to obtain considerable signal and contrast enhancements in the detection of the specific interaction between the antibody and the cotinine in the fingerprint (Fig. 4). The citrate reducing and stabilizing layer on the gold nanoparticle surface is replaced by protein A linked by a bifunctional thiolate linker $\mathrm{N}$-succinimidyl 3-(2-pyridyldithio)propionate to facilitate the binding of the protein on the surface of gold nanoparticles. The anticotinine antibody is assembled onto the protein A monolayer surrounding the gold nanoparticles. The anticotinine-nanoparticle conjugates were applied to the detection of cotinine in the fingerprints of smokers. The typical images obtained show that a partial ridge pattern of a fingerprint appears to be discernable when the fingerprints are fluorescently imaged by using the anticotinine antibody. Such images do indicate the presence of cotinine, confirming that the individual is a smoker [40].

\section{Vacuum metal deposition}

Vacuum metal deposition (VMD) is a long-established industrial technique for the deposition of metal coatings to surfaces such as glass to form a mirror [41, 42]. Research was also conducted into the ability of the technique to detect latent prints on fabrics. These experiments showed that although some print development was obtained by the use of single metals (e.g., gold, silver, copper, zinc, and cadmium), the best results were obtained by the use of a combination of metals, typically gold or silver followed by cadmium or zinc (Fig. 5). Initially, the gold and cadmium combination was selected; but due to health concerns, the gold and zinc combination was being recommended instead. Gold was preferred over silver as the initial deposition metal because silver can be degraded by fingerprint secretions.

There is a great variability in the speed at which different substrates coat, and it may take more than $10 \mathrm{~min}$ to obtain a suitable coating on some types of materials. In some cases, it may be necessary to carry out multiple deposition runs in order to obtain satisfactory results. The VMD technique was initially adopted as an operational technique for the detection of latent prints on thin polyethylene sheets. Recently this technique has been utilized in non-porous fabrics such as nylon and cotton [43]. Polymer type, among other factors, and previous treatment of the print with cyanoacrylate will affect the VMD conditions required for optimum development [44].

\section{Future prospects for gold in forensic science}

The progress in forensic chemistry is slowed by the on-site demand for fast and efficient conclusion. Gold chemistry contributed to the fingerprint detection utilizing the singlemetal and multi-metal deposition techniques. This field can be expanded to serve the need for more materials in illicit drug detection as well, although slow progress has been made already. The detection of explosives and nerve gases on the crime scene is of current interest. The photophysical properties of gold are not yet utilized in this field. In search for compounds for the detection of explosives, fluorescence quenching-based detection of explosives represents one of the most promising methods. Quenching fluorescent gold complexes by nitroaromatics can be used as a chemical signature of explosives.

Open Access This article is distributed under the terms of the Creative Commons Attribution License which permits any use, distribution and reproduction in any medium, provided the original author(s) and source are credited.

\section{References}

1. Corti C, Holliday R (2010) Gold: science and applications. CRC, Boca Raton, FL, pp 161-189

2. Mohr F (ed) (2009) Gold chemistry: highlights and future directions. Wiley-VCH, Weinheim

3. Mohamed AA (2010) Advances in the coordination chemistry of nitrogen ligand complexes of coinage metals. Coord Chem Rev 254:1918-1947

4. Fackler JP (2002) Forty-five years of chemical discovery including a golden quarter-century. Inorg Chem 41:6959-6972

5. Hashmi ASK, Toste DF (eds) (2009) Modern gold catalyzed synthesis. Wiley-VCH Verlag $\mathrm{GmbH}$, Weinheim

6. Bond GC, Louis C, Thompson DT (2006) Catalysis by gold (Catalytic Science Series, vol. 6). Imperial College Press, London

7. Laguna A (ed) (2008) Modern Supramolecular gold chemistry: goldmetal interactions and applications. Wiley, Weinheim, Germany

8. Stimson PG, Mertz CA (eds) (1997) Forensic dentistry. CRC, Boca Raton, FL

9. Saferstein R (2011) Criminalistics: an introduction to forensic science, 10th edn. Prentice Hall, Englewood Cliffs, NJ

10. Newton DE (2007) Forensic chemistry. Facts on file library

11. Lee HC, Gaensslen RE (eds) (2001) Advances in fingerprint technology, 2nd edn. Boca Raton, CRC press

12. Bell SC, Oldfield LS, Shakleya DM, Petersen JL, Mercer JW (2006) Chemical composition and structure of the microcrystals formed between silver(I) and $\gamma$-hydroxybutyric acid and $\gamma$ hydroxyvaleric acid. J Forensic Sci 51:808-811 
13. Choi MJ, McDonagh AM, Maynard P, Roux C (2008) Nanotechnology gold forensic metal-containing nanoparticles and nanostructured particles in fingermark detection. Forensic Sci Int 179:87-97

14. Smith FP (ed) (2005) Handbook of forensic drug analysis. Elsevier Academic Press, Amsterdam

15. Terminology and Information on Drugs, 2nd Edition, United Nations, NY, (2003) http://www.unodc.org/unodc/en/illicit-drugs/ definitions

16. Recommended methods for the identification and analysis of amphetamine, methamphetamine and their ring-substituted analogues in seized materials. United Nations, NY (2006) http:// www.unodc.org/

17. Color test reagents kits for preliminary identification of drugs of abuse. National law enforcement and corrections technology center NIJ standard-0604.01, Washington DC (2000) http://www. justnet.org/

18. American Society for Testing and Materials (2003) ASTM E196898. Standard guide for microcrystal testing in the forensic analysis of cocaine-14.02. West Conshohocken, Pennsylvania: ASTM International

19. Lyons AB (1885) Am J Pharm 30:465-477

20. Wood MR, Brettell TA, Lalancette RA (2007) The gold(III) tetrachloride salt of L-cocaine. Acta Crystallogr C63:m33-m35

21. Wood MR, Thompson HW, Brettell TA, Lalancette RA (2010) The hydrated and anhydrous gold(III) tetrachloride salts of Lecgonine, an important forensic toxicology marker for cocaine. Acta Crystallogr C66:4-8

22. Abelardo AV, Nora RM (1966) New microcrystalline reactions for the identification of cocaine, heroin, morphine, and codeine. Archivos de Bioquimica, Quimica y Farmacia 13:73-77

23. Allen AC, Cooper DA, Kiser WO, Cottrell RC (1981) The cocaine diastereoisomers. J Forensic Sci 26:12-26

24. Fulton CC (1969) Modern microcrytal tests for drugs: the identification of organic compounds by microcrystalloscopic chemistry. Wiley, New York, pp 300-301

25. Isenschmid DS, Levine BS, Caplan YH (1989) A comprehensive study of the stability of cocaine and its metabolites. J Anal Toxicol $13: 250-256$

26. Klingmann A, Skopp G, Aderjan R (2001) Analysis of cocaine, benzoylecgonine, ecogonine methyl ester, and ecgonine by highpressure liquid chromatography-API mass spectrometry and application to a short-term degradation study of cocaine in plasma. J Anal Toxicol 25:425-430

27. Logan BK (2001) Ecgonine is an important marker for cocaine use in inadequately preserved specimens. J Anal Toxicol 25:219 220

28. Ashbaugh DR (1999) Quantitative-qualitative friction ridge analysis: an introduction to basic and advanced ridgeology. CRC Press, Boca Raton, Florida

29. Lobitz WC, Mason HL (1948) Chemistry of palmar sweat: VII. Discussion of studies on chloride, Urea, Glucose, Uric Acid,
Ammonia-Nitrogen, and Creatinine. Arch Dermatol Syphilol 57:907-915

30. Ruhemann S (1910) Cyclic di- and tri-ketones. J chem Soc Trans 97:1438-1449

31. Cantu AA (2001) Silver physical developers for the visualization of latent prints on paper. Forensic Sci Rev 13:30

32. Becue A, Champod C, Margot P (2007) Use of gold nanoparticles as molecular intermediates for the detection of fingerprints. Forensic Sci Int 168:169-176

33. Gao D, Li F, Songa J, Xu X, Zhang Q, Niu L (2009) One step to detect the latent fingermarks with gold nanoparticles. Talanta 80:479-483

34. Becue A, Scoundrianos A, Champod C, Margot P (2008) Fingermark detection based on the in situ growth of luminescent nanoparticles - towards a new generation of multimetal deposition. Forensic Sci Int 179:39-43

35. Stauffer E, Becue A, Singh KV, Thampi KR, Champod C, Margot $P$ (2007) Single-metal deposition (SMD) as a latent fingermark enhancement technique: an alternative to multimetal deposition (MMD). Forensic Sci Int 168:e5-e9

36. Sametband M, Shweky I, Banin U, Mandler D, Almog J (2007) Application of nanoparticles for the enhancement of latent fingerprints. Chem Commun 11:1142-1144

37. Hussain I, Hussain SZ, Habib-ur-Rehman AI, Rehman A, Khalid ZM, Brust M, Cooper AI (2010) In situ growth of gold nanoparticles on latent fingerprints-from forensic applications to inkjet printed nanoparticle patterns. Nanoscale 2:2575-2578

38. Sodhi GS, Kaur J (2001) Powder method for detecting latent fingerprints. Forensic Sci Int 120:172-176

39. Choi MJ, McDonagh AM, Maynard PJ, Wuhrer R, Lennard C, Roux C (2006) Preparation and evaluation of metal nanopowders for the detection of fingerprints on non-porous surfaces. J Forensic Identif 56:756-768

40. Leggett R, Lee-Smith EE, Jickells SM, Russell DA (2007) "Intelligent" fingerprinting: simultaneous identification of drug metabolites and individuals by using antibody-functionalized nanoparticles. Angew Chem Int Ed 46:4100-4103

41. Theys P, Turgis Y, Lepareux A, Chevet G, Ceccaldi PF (1968) New technique for bringing out latent fingerprints on paper: vacuum metallisation. Int Crim Police Rev 217:106

42. Kent $\mathrm{T}$ (1982) User guide to the metal deposition process for the development of latent fingerprints. Home Office Scientific research and development branch (HOSRDB), Aldermaston, England

43. Fraser J, Sturrock K, Deacon P, Bleay S, Bremner DH (2010) Visualisation of fingermarks and grab impressions on fabrics. Part 1: Gold/zinc vacuum metal deposition. Forensic Sci Int. doi:10.1016/j.forsciint.2010.11.003

44. Jones N, Mansour D, Stoilovic M, Lennar C, Roux C (2001) The influence of polymer type, print donor and age on the quality of fingerprints developed on plastic substrates using vacuum metal deposition. Forensic Sci Int 124:167-177 